\title{
Sono, Qualidade de Vida e Depressão em Mulheres no Pós-tratamento de Câncer de Mama
}

\author{
Sleep, Quality of Life and Depression in Women in Breast Cancer Post-Treatment
}

\author{
Renatha El Rafihi-Ferreira ${ }^{a}$, Maria Laura Nogueira Pires ${ }^{b}, \&$ Maria Rita Zoéga Soares ${ }^{*}, a$ \\ ${ }^{a}$ Universidade Estadual de Londrina, Londrina, Brasil \\ $\&{ }^{b}$ Universidade Estadual Paulista Júlio de Mesquita Filho, Assis, Brasil
}

\begin{abstract}
Resumo
Este trabalho investigou a qualidade de sono de mulheres com câncer de mama e suas relações com qualidade de vida e depressão. Cinqüenta mulheres com câncer de mama (grupo clínico) e cinqüenta controles responderam um questionário médico-demográfico e ao instrumento Pittsburgh Sleep Quality Index. O grupo clínico também respondeu aos instrumentos Quality of Life Cancer-Survivor e Brief Zung SelfRating Depression Scale. Verificou-se que mulheres com câncer tinham significativamente mais queixas de nictúria, calor e despertares noturnos. O grupo clínico com má qualidade do sono apresentou comprometimento na qualidade de vida e mais sintomas de depressão. Em geral, a qualidade de sono em mulheres com câncer de mama pode predizer a qualidade de vida e o bem estar psicológico.

Palavras-chave: Câncer de mama, insônia, qualidade de vida, depressão.

Abstract

This study investigated the sleep quality of women with breast cancer and their relationship with quality of life and depression. Fifty women with breast cancer (clinical group) and a control group of other 50 women without the disease answered a clinical-demographic questionnaire and the Pittsburgh Sleep Quality Index. The clinical group also completed the Quality of Life Cancer-Survivor and the Brief Zung SelfRating Depression Scale questionnaires. It was found that women with breast cancer had significantly more complaints of nocturia, heat and nighttime awakenings. The clinical group with poor quality of sleep reported impaired quality of life and more symptoms of depression. In general terms, sleep quality in women with breast cancer can predict their quality of life and psychological well-being.

Keywords: Breast cancer, insomnia, quality of life, depression.
\end{abstract}

Queixas de sono, particularmente insônia, são freqüentes em pacientes com câncer de mama, com prevalências que superam as encontradas na população geral. Os resultados sumarizados em recente revisão sobre o tema e estudos complementares mostram que a prevalência da insônia, tanto na hospitalização, quanto ao longo do tratamento ou depois, pode variar entre $23 \%$ e $80 \%$ (Bardwell et al., 2008; Fiorentino \& Ancoli-Israel, 2006; Furlani \& Ceolin, 2006). Contudo, diferentemente da atenção que

" Este artigo foi parte da dissertação de mestrado da primeira autora, sob orientação da terceira autora. Este trabalho recebeu apoio financeiro do Conselho Nacional de Desenvolvimento Científico e Tecnológico ([CNPq], Processo 477639/2008-9). A primeira autora foi bolsista da Coordenação de Aperfeiçoamento de Pessoal de Nível Superior (CAPES)

Endereço para correspondência: Departamento de Psicologia Geral e Análise do Comportamento, Universidade Estadual de Londrina, Rodovia Celso Garcia Cid, PR 445, KM 380, Caixa Postal 6001, Londrina, PR, Brasil 86051-990. Tel./Fax: (43) 3371-4227. E-mail: ritazoega@hotmail.com recebem outras manifestações físicas ou psicológicas que acometem o paciente oncológico, como fadiga, dor, náusea, depressão e ansiedade, problemas de sono são vistos como uma reação esperada e temporária, advinda de circunstâncias estressoras que circundam o diagnóstico (impacto emocional, ansiedade, depressão, sensação de incerteza) e do tratamento (hospitalização, procedimentos cirúrgicos, efeitos colaterais de medicação como náusea e vômito). No entanto, embora tal miríade de estressores seja o evento precipitante, a dificuldade em obter um sono de boa qualidade e de duração adequada pode se perpetuar em decorrência do estabelecimento de hábitos inadequados de sono em resposta a eles (Savard \& Morin, 2001). Assim, freqüentemente, pacientes oncológicos passam períodos prolongados na cama, cochilam em vários momentos ao longo do dia, e têm horários irregulares para dormir e acordar (O’Donnell, 2004).

Contudo, sobre os reconhecidos aspectos negativos da insônia, Savard e Morin (2001) salientam que os próprios pacientes minimizam suas dificuldades com o sono, relativizando sua importância em comparação aos demais 
aspectos relacionados ao câncer, os quais julgam mais importantes para seu próprio bem estar. Também, freqüentemente os profissionais da saúde envolvidos nos cuidados ao paciente oncológico vêem a insônia como um sintoma secundário à depressão ou ansiedade, cujo alívio estaria então na dependência da resolução dessas condições primárias. Ainda, de acordo com Savard e Morin (2001), há um conhecimento insuficiente, por parte da equipe de saúde, sobre aspectos relacionados ao diagnóstico e opções de tratamento da insônia, o que torna o cenário ainda carente de atenção.

A despeito das causas e conseqüências da insônia, pesquisas mostram uma forte associação entre problemas de sono e distúrbios psiquiátricos e médicos (Stepanski, 2006). Sobre essa questão, estudos (Buysse, Angst, et al., 2008; Fleming, Gillespie, \& Espie, 2010; Hindi, Dew, Albert, Lotrich, \& Reynolds, 2011; Lustberg \& Reynolds, 2000; Rosekind \& Gregory, 2010) apontam a insônia crônica como conseqüência e fator de risco para o desenvolvimento ou complicação da depressão. Lustberg e Reynolds (2000) salientam que o sono é fisiologicamente anormal em pessoas com risco para o distúrbio de humor, com reduzida latência do sono REM não só durante os episódios depressivos. Assim, a persistência da insônia pode indicar crescente vulnerabilidade a recaida ou recorrência depressiva em indivíduos preveamente recuperados (Lustberg \& Reynolds, 2000; Stepanski, 2006). Tal questão torna-se mais complexa ao considerar pessoas com diagnóstico de câncer. Autores (Kleiboer et al., 2011; McChargue et al., 2010; Reich, Lesur, \& Perdrizet-Chevallier, 2008) apontam alta prevalência de sintomas depressivos em pessoas durante e após o tratamento oncológico. Segundo Fleming et al., (2010) os indivíduos com câncer freqüentemente desenvolvem distúrbios de sono após o diagnóstico, que geralmente evolui para insônia crônica, resultando em diminuição na qualidade de vida, prejuízos no humor e redução de energia.

Embora distúrbios de sono em pacientes oncológicos seja um tema de relevância as informações sobre essa população específica ainda são limitadas quando comparadas com o conhecimento acumulado sobre a insônia e seus impactos na população geral. No Brasil, em particular, a associação entre câncer e problemas de sono é assunto relativamente recente, com estudos em mulheres com câncer ginecológico e mamário em período de hospitalização (Furlani \& Ceolin, 2006), em pacientes em tratamento com câncer urinário, de cabeça e pescoço (Barichello, Sawada, Sonobe, \& Zago 2009) e em adolescentes sobreviventes de câncer infantil (Silva, 2009). Visando ampliar a compreensão sobre o tema, este estudo teve os seguintes objetivos: (a) investigar a qualidade de sono de mulheres em pós-tratamento de câncer de mama e compará-las com um grupo controle; (b) investigar a relação entre qualidade de sono, qualidade de vida e depressão em mulheres no pós-tratamento de câncer de mama.

\section{Material e Método}

Trata-se de estudo do tipo transversal, exploratório e descritivo.

\section{Participantes}

Fizeram parte do estudo cinqüenta mulheres adultas com diagnóstico prévio de câncer de mama (Estágio I ou II), que se encontravam entre um a dez anos de pós-tratamento (cirurgia, quimioterapia e/ou radioterapia) e cinqüenta mulheres adultas sem diagnóstico prévio ou atual de qualquer tipo de câncer e com resultados negativos em testes de mamografia. O critério de exclusão utilizado foi à presença de encefalopatia (aguda ou crônica) e distúrbios psiquiátricos graves, suficientes para impedir a participação no estudo.

As participantes do grupo clínico se encontravam em pós-tratamento de quimioterapia (66\%) e/ou radioterapia (54\%) e/ou cirurgia (40\%). De acordo com auto-relato e prontuários médicos todas as participantes estavam livres da enfermidade. O período de pós-tratamento variou de 1 a 10 anos (42\% entre um a dois anos, $40 \%$ entre 3 a 5 anos e $18 \%$ de 6 a 10 anos) com média de 46,02 meses e $D P=33,1$. A idade do grupo clínico foi significativamente superior em relação ao grupo controle $(48,5 \pm 12,9$ vs $55,5 \pm 11,5 ; t=2,9, p<0,05)$. Metade do grupo clínico e $56 \%$ do grupo controle tinham nível superior $(Z=0,6 ; n s)$, sendo a maioria casada ( $66 \%$ vs $58 \% ; Z=0,8 ; \mathrm{ns})$. A proporção de mulheres que exercia atividade remunerada foi aproximadamente duas vezes menor no grupo clinico em comparação ao controle (42 vs 76, $Z=3,5 ; p<0,05$ ). Metade das participantes do grupo clínico estava em tratamento hormonal, principalmente com tamoxifeno ( $77 \%$ dos casos). Em contrapartida, no grupo controle apenas $8 \%$ relataram fazer tratamento hormonal, sendo o estradiol o mais utilizado $(N=3)$. A maioria $(90 \%)$ do grupo clínico estava na menopausa, sendo que $69 \%$ dessas mulheres entraram na menopausa devido ao tratamento oncológico.

\section{Instrumentos}

Todos os instrumentos foram traduzidos por um profissional qualificado, sob acompanhamento e supervisão das autoras do artigo.

Questionário sobre Informação Médica-Demográfica. Composto por itens relacionados às informações médicas (situação e estágio da enfermidade, medicação, terapias, inclusive terapia oncológica atual e medicação para cuidados paliativos) e demográficas - idade, escolaridade, estado civil, atividade remunerada (Passik, 2007).

Pittsburgh Sleep Quality Index (PSQI). O PSQI tem um bom índice de confiabilidade e validade e têm sido amplamente utilizado em estudos psiquiátricos, médicos gerais e também em estudos oncológicos (Barichello et al., 2009; Beck et al., 2010; Buysse, Hall, et al., 2008; Buysse, Reynolds, Monk, Berman, \& Kupfer, 1989; 
Carlson, Campbell, Garland, \& Grossman, 2007; Fortner, Stepanski, Wang, Kasprowicz, \& Durrence, 2002; Furlani \& Ceolin, 2006). O uso do instrumento no Brasil não é recente, e as propriedades psicométricas da sua versão em português foram objeto de investigação (Barichello et al., 2009; Bertolazi et al., 2011; Cardoso et al., 2009; Furlani \& Ceolin, 2006). Este instrumento é composto por sete áreas relacionadas ao sono (qualidade subjetiva, latência, duração, eficiência habitual, distúrbios, uso de medicação para dormir e sonolência diurna) que resultam em um escore (de 0 a 21) correspondente a qualidade global de sono. Estabelecido pela escala original, a obtenção de até 5 pontos indica boa qualidade de sono, e acima de 5 pontos, má qualidade de sono (Buysse et al., 1989). A utilização do ponto de corte de 5 pontos resultou numa especificidade de $86,5 \%$ e sensibilidade de $89,6 \%$ na identificação de casos com distúrbios de sono diagnosticados por meio de critérios clínicos, questionários e registros polissonográficos (Buysse et al., 1989). Recentemente, Buysse, Hall, et al. (2008) demonstraram que subgrupos categorizados por meio desse critério também diferem entre si em relação a variáveis de sono obtidas por meio de registros diários e em medidas de auto-relato de sintomas psicológicos. O ponto de corte 5 é amplamente utilizado em estudos (Barichello et al., 2009; Beck et al., 2010; Carlson et al., 2007; Fortner et al., 2002; Furlani \& Ceolin, 2006) que investigam qualidade de sono em pacientes com câncer. $\mathrm{O}$ coeficiente alfa de Cronbach foi utilizado para avaliar a consistência interna do instrumento, que no presente estudo obteve um valor de 0,72 , um valor próximo à variação observada (de 0,77 a 0,83) em outros estudos (Beck, Schwartz, Towsley, Dudley, \& Barsevick, 2004; Bertolazi et al., 2011; Buysse et al., 1989; Carpenter \& Andrykowski, 1998; Doi et al., 2000).

Quality of Life Cancer-Survivor (QOL-CS). Este instrumento vem sendo amplamente utilizado na descrição da qualidade de vida de pessoas que encontram-se especificamente em pós tratamento de câncer (Dis, Mols, Vingerhoets, Ferrell, \& Poll-Franse, 2006; Ferrell, Dow, \& Grant, 1995; Zebrack \& Chesler, 2001). É composto por 41 itens que são divididos em quatro sub-escalas, físico (8 itens), psicológico (18 itens), social (8 itens) e espiritual ( 7 itens). Os itens são respondidos por meio de uma escala que varia de 0 (pior resultado) a 10 - melhor resposta (Ferrell et al., 1995). As quatro sub-escalas do QOL-CS obtiveram os seguintes alfas de Conbrach neste estudo: bem estar físico $=0,81$; bem estar psicológico $=0,82$; bem estar social $=0,73$; bem estar espiritual $=0,56$. O escore global da escala obteve um alfa de 0,70 . Os resultados obtidos na maioria das sub-escalas foram satisfatórios e semelhantes aos valores relatados em outros estudos (Dis et al., 2006; Ferrell et al., 1995; Zebrack \& Chesler, 2001). Nesse contexto, vale mencionar que um baixo valor de alfa para a sub-escala bem estar espiritual foi também observado por Dis et al. (2006).
Brief Zung Self-Rating Depression Scale (BZSDS). O instrumento avalia a presença de sintomas depressivos. Constitui-se de uma versão reduzida (11 itens) do instrumento Zung Self-Rating Depression Scale, que omite nove itens relativos aos sintomas somáticos. Esta versão resumida foi projetada para maximizar a confiabilidade da identificação dos sintomas cognitivos da depressão (por exemplo, anedonia,concentração, disforia, desesperança), ao eliminar os sintomas somáticos que muitas vezes são confundidos com o tratamento do câncer - por exemplo, fadiga, alterações do apetite e insônia (Passik et al., 2001). Os itens são respondidos por meio de uma escala de 1 a 4 . Escores entre 22 a 32 são indicativos de depressão com gravidade leve, entre 33 a 38 com gravidade moderada e entre 39 a 44 com gravidade severa (Passik et al., 2001). No atual estudo, o coeficiente alfa de Cronbach foi de 0,82 , valor similar ao relatado $(0,84)$ por Dugan et al. (1998) em pacientes com câncer.

\section{Procedimento}

As participantes foram identificadas pelo médico responsável de uma clínica de ginecologia/mastologia e encaminhadas para participação na pesquisa. As mulheres de ambos os grupos foram contatadas via telefone pela pesquisadora e convidadas a participar do estudo. A coleta dos dados foi agendada em um encontro individual com dia e horário de acordo com a disponibilidade das participantes. Por escolha delas, tal coleta de dados ocorreu em suas residências. No encontro as mulheres assinaram o Termo de Consentimento Livre e Esclarecido, e foram informadas sobre os objetivos da pesquisa e de que poderiam ter acesso aos dados após a conclusão do estudo. A pesquisadora aplicou o questionário sobre informação médica-demográfica e o PSQI em todas as participantes. Os instrumentos QOL-CS e BZSDS foram aplicados somente nas mulheres do grupo clínico. Durante a aplicação dos instrumentos, grande parte das participantes do grupo clínico verbalizou sentimentos e lembranças, indicando a evocação de aspectos emocionais relacionadas à doença. Ao final da pesquisa, o mastologista recebeu uma listagem de nomes de mulheres que foram identificadas como tendo má qualidade de sono para posterior avaliação. Este estudo recebeu aprovação do Comitê de Ética em Pesquisa Envolvendo Seres Humanos da Universidade Estadual de Londrina/ Hospital Universitário Regional Norte do Paraná (Protocolo SISNEP $n^{\circ} 210 / 09$ ).

\section{Análise dos Dados}

Foram realizadas análises descritivas (proporções, médias e desvios padrões). Diferenças entre proporções foram analisadas usando o teste $Z$. Comparações entre dois grupos foram realizadas por meio do teste $t$ e pelo cálculo da magnitude do efeito. A magnitude do efeito, expressa como $d$, foi estimada como a diferença entre as médias dos grupos com boa e má qualidade de sono divi- 
Rafihi-Ferreira, R., Pires, M. L. N. \& Soares, M. R. Z. (2012). Sono, Qualidade de Vida e Depressão em Mulheres no Pós-tratamento de Câncer de Mama.

dida pelo desvio padrão comum. Convencionalmente, um valor de $d=0,20$ representa uma magnitude de efeito pequeno, $d=0,50$ indica magnitude média e $d=0,80$ indica uma magnitude de efeito elevada (Cohen, 1988). Comparações entre quatro grupos foram feitas por meio de análise de variância de duas vias, seguido pelo teste de Newman-Keuls quando apropriado. Foram realizadas análises de regressão para verificar a capacidade preditiva do sono para qualidade de vida e depressão. Um valor de $p<0,05$ foi considerado significante.

\section{Resultados}

Os resultados foram divididos em: (a) Queixas relacionadas ao sono; (b) Qualidade de sono em mulheres com câncer de mama; (c) Sono e qualidade de vida; (c) Sono e depressão; e (d) Análise da qualidade de sono como predição para qualidade de vida e depressão.

\section{Queixas Relacionadas ao Sono}

A Tabela 1 refere-se à proporção de mulheres de ambos os grupos que relataram queixas relacionadas ao sono com freqüência igual ou superior a três vezes na semana.

Tabela 1

Freqüencias de Mulheres que Referiram Queixas de Sono nos Grupos Controle $(N=50)$ e Clínico $(N=50)$

\begin{tabular}{lccc}
\hline \multicolumn{1}{c}{ Queixas } & Controle (\%) & Clínico (\%) & $Z$ \\
\hline Acordar para ir ao banheiro & 26 & 52 & $2,7^{*}$ \\
Acordar no meio da noite ou muito cedo & 22 & 40 & $1,9^{*}$ \\
Sentir muito calor & 14 & 36 & $2,5^{*}$ \\
Dor & 14 & 20 & 0,8 \\
Tossir ou roncar forte & 16 & 16 & 0 \\
Dificuldades para respirar & 8 & 8 & - \\
Sentir muito frio & 6 & 4 & - \\
Medicação para dormir & 12 & 16 & 0,6 \\
Sonolência diurna & 2 & 4 & - \\
\hline
\end{tabular}

$* p<0,05$.

Constata-se que um número elevado e comparável de mulheres do grupo controle e do clínico referiu demorar mais de trinta minutos para adormecer $(42 \%$ vs $38 \%$, $Z=0,4$, ns), tinha duração de sono inferior a seis horas $(18 \%$ vs $14 \%, 0,6, \mathrm{~ns})$ e avaliou a qualidade de seu sono como ruim ou muito ruim $(22 \%$ vs $26 \%, 0,5, \mathrm{~ns})$. Todas as participantes, do grupo clínico e controle indicaram alguma queixa relacionada ao sono, identificadas pelo PSQI. Contudo $74 \%$ do grupo clínico e $50 \%$ do grupo controle apresentaram em freqüência igual ou superior a três dias na semana. Despertar para ir ao banheiro, acordar no meio da noite ou muito cedo e sentir calor foram mais freqüentemente referidos por mulheres do grupo clínico. Não houve diferença entre os grupos na freqüência com que relata- ram queixas de dor, tossir ou roncar forte, dificuldades para respirar, sentir muito frio e sonolência diurna. $\mathrm{O}$ uso crônico de medicação foi relatado em proporções semelhantes em ambos os grupos, que apontaram nunca terem procurado e/ou recebido qualquer tipo de tratamento não farmacológico para a melhoria da qualidade de sono.

A soma da pontuação de todos os componentes do PSQI permitiu classificar as participantes com boa e má qualidade de sono.

\section{Qualidade de Sono em Mulheres com Câncer de Mama}

A Tabela 2 demonstra os padrões de sono para as participantes dos grupos clínico e controle, classificadas com boa e má qualidade de sono.

Tabela 2

Padrões de Sono de Mulheres dos Grupos Controle e Clínico Classificadas com Boa ou Má Qualidade de Sono por Meio do PSQI

\begin{tabular}{lrrrrrrrrr}
\hline & \multicolumn{4}{c}{ Controle } & \multicolumn{4}{c}{ Clínico } \\
& Boa $(n=25)$ & \multicolumn{2}{c}{ Má $(n=25)$} & \multicolumn{2}{c}{ Boa $(n=30)$} & Má $(n=20)$ & \\
& $M$ & $D P$ & $M$ & $D P$ & $M$ & $D P$ & $M$ & $D P$ & $F_{(1,96)}$ \\
\hline Horário de deitar (h) & 23,1 & 1,3 & 23,8 & 1,3 & 23,0 & 1,0 & 23,1 & 0,8 & 2,1 \\
Latência (min) & 19,1 & 19,5 & 30,0 & 25,2 & 12,3 & 11,9 & 46,3 & 41,4 & $5,1^{*}$ \\
Horário de acordar (h) & 6,8 & 1,2 & 7,0 & 1,3 & 6,9 & 1,0 & 6,8 & 1,2 & 0,4 \\
Duração do sono (h) & 7,4 & 1,0 & 6,8 & 1,5 & 7,7 & 1,1 & 6,9 & 1,9 & 0,01 \\
Eficiência do sono (\%) & 95,2 & 5,0 & 92,8 & 7,3 & 97,4 & 2,5 & 88,6 & 12,4 & $4,9^{*}$ \\
\hline
\end{tabular}

$* p<0,05$. 
Usando o ponto de corte estabelecido por Buysse et al. (1989), os resultados revelaram que $40 \%$ das participantes com câncer de mama e $50 \%$ das mulheres do grupo controle apresentaram má qualidade de sono. Em comparação ao grupo controle, as participantes do grupo clínico com má qualidade de sono apresentaram latência do sono significativamente maior e uma menor eficiência do sono. Os grupos não diferiram entre si nas demais variáveis. A análise individual das respostas ao PSQI revelou que $30 \%$ do grupo clínico e $20 \%$ do grupo controle relataram latência de sono superior a 60 minutos.
Para comparar a qualidade de sono com os resultados dos instrumentos que avaliaram qualidade de vida e depressão, os escores do QOL-CS e BZSDS das participantes do grupo clínico foram divididos entre as mulheres com boa e má qualidade de sono, segundo a medida apontada pelo PSQI.

\section{Sono e Qualidade de Vida}

A Tabela 3 indica os escores dos quatro domínios do QOL-CS obtidos nas participantes do grupo clínico com boa e má qualidade de sono.

\section{Tabela 3}

Escores no QOL-CS das Mulheres do Grupo Clínico Classificadas com Boa ou Má Qualidade de Sono por Meio do PSQI

\begin{tabular}{llllllc}
\hline \multicolumn{1}{c}{ Itens } & \multicolumn{2}{c}{ Boa $(n=30)$} & \multicolumn{2}{c}{$\operatorname{Má}(n=20)$} & & \\
& $M$ & $D P$ & $M$ & $D P$ & $t_{(48)}$ & $d$ \\
\hline Total Sub-escala Física & 8,3 & 0,6 & 6,1 & 1,7 & $4,1^{*}$ & 1,8 \\
Total Sub-escala Psicológica & 6,3 & 2,0 & 4,9 & 2,1 & $3,3^{*}$ & 0,7 \\
Total Sub-escala Social & 7,4 & 1,8 & 5,8 & 1,8 & $3,2^{*}$ & 0,9 \\
Total Sub-escala Espiritual & 7,2 & 2,0 & 6,2 & 2,3 & 1,9 & \\
\hline TOTAL GERAL & 7,0 & 1,9 & 5,5 & 2,0 & $4,7^{*}$ & 0,8 \\
\hline
\end{tabular}

Nota. Escore $0=$ piores resultados, escore $10=$ melhores resultados. $* p<0,05$.

A análise estatística mostrou que as participantes com má qualidade do sono referiram maior comprometimento nos domínios físico, psicológico e social, resultando em valores de magnitude de efeito elevados (valores de $d$ entre 0,7 e 1,8). Em relação ao domínio físico, mulheres com má qualidade de sono referiram piores pontuações nos itens que avaliam fadiga, mal estar ou dores, qualidade de sono, constipação intestinal e saúde física geral. Quanto ao domínio psicológico, estas mulheres referiram maior comprometimento nos itens enfrentamento, depressão e ansiedade. No domínio social, este grupo relatou maior prejuízo nos itens referentes aos relacionamentos pessoais, solidão e aspectos financeiros. Embora a ausência de diferenças significativas quanto ao domínio espiritual, mulheres com má qualidade de sono referiram maior incerteza em relação ao futuro.

\section{Sono e Depressão}

A avaliação dos comportamentos depressivos pelo BZSDS mostrou que as mulheres do grupo com má qualidade de sono obtiveram uma pontuação média significativamente maior do que as mulheres do grupo com boa qualidade de sono $\left(20,8 \pm 7,12\right.$ vs $16,6 \pm 3,76 ; t_{(\mathrm{gl}=48)}=-$ $2,69, p<0,05 ; d=0,7)$. A freqüência de mulheres com comportamento depressivo com gravidade leve foi significativamente maior no grupo com má qualidade de sono do que entre aquelas que dormiam bem ( $40 \%$ vs $13 \% ; Z=2,63$, $p=0,03)$. O comportamento depressivo com gravidade moderada ocorreu somente entre aquelas do grupo com má qualidade de sono (5\%).

\section{Análise Complementar: Análise de Regressão}

As análises de regressão demonstraram que a qualidade de sono significativamente prediz os escores de qualidade de vida, $\beta=-0,18 ; t(48)=-4,1 ; p<0,05$ e os escores de depressão, $\beta=0,60 ; t(48)=3,1 ; p<0,05$. Também explica uma proporção significativa da variância nos escores de qualidade de vida, $R^{2}=0,25 ; F(1,48)=17,13 ; p<0,05$ e nos escores de depressão, $R^{2}=0,15 ; F(1,48)=9,89 ; p<0,05$.

\section{Discussão}

Este estudo demonstra uma particularidade ao selecionar instrumentos específicos para avaliação da qualidade de sono e suas relações com qualidade de vida e depressão em mulheres no pós-tratamento de câncer de mama. O conjunto específico desses instrumentos não foi utilizado anteriormente em outros estudos publicados. Além disso, no Brasil não haviam estudos que avaliaram a qualidade de sono no pós-tratamento de câncer de mama e que relacionaram a qualidade de sono com qualidade de vida e depressão. Neste sentido, o presente estudo buscou uma contribuição adicional.

Esta pesquisa demonstra que participantes de ambos os grupos apresentaram problemas com sono. $\mathrm{O}$ fato de metade das participantes do grupo controle serem classificadas por meio do PSQI com má qualidade de sono corrobora os dados apresentados na literatura, que salientam que $30 \%$ a $40 \%$ das mulheres da população geral relatam sintomas de insônia (Santos-Silva et al., 2010). Além disso, os dados demonstram dificuldades relacionadas ao 
sono entre as mulheres que terminaram o tratamento oncológico. De fato, $40 \%$ do grupo clínico relataram dificuldades relacionadas ao sono medidas pelo PSQI, caracterizado por uma latência de sono prolongada, sono fragmentado em decorrência de freqüentes despertares devido ao calor, dor, tosse, nictúria ou por despertarem antes do momento desejado. Essas queixas foram semelhantes às relatadas por Fortner et al. (2002) e Furlani e Ceolin (2006), embora grande parte das participantes destes estudos estavam em tratamento. Comparado ao grupo controle, as mulheres do grupo clínico despertavam em maior freqüência devido ao calor e nicturia.

O despertar várias vezes durante a noite, por decorrência de calor ou por nictúria, pode estar associado ao fato de que a maioria das participantes estava menopausadas devido ao tratamento oncológico. Eichling e Sahni (2005) apontam que os sintomas vasomotores são os principais preditores das perturbações do sono que freqüentemente acompanham a menopausa. De maneira geral, segundo esses autores, os sintomas vasomotores são as primeiras alterações que surgem no climatério e também são os que mais conturbam o bem-estar da mulher. Nesta fase é comum as mulheres terem menor eficiência do sono e mais queixas relacionadas à sua qualidade, possivelmente em decorrência dos calores noturnos e nictúria (Campos, Bittencourt, Haidar, Tufik, \& Baracat, 2005; Soares, 2006).

Além da alta freqüência de dificuldades com o sono, os resultados mostraram que o sono pode predizer a qualidade de vida. Em consonância com a literatura (Davidson, MacLean, Brundage, \& Schulze, 2002; Fiorentino \& Ancoli-Israel, 2006; Fortner et al., 2002; Furlani \& Ceolin, 2006; Koopman et al., 2002; Palesh et al., 2007) mulheres com câncer de mama com má qualidade de sono relataram prejuízos na qualidade de vida relacionada a aspectos físicos como saúde física geral, fadiga, mal estar, dor e constipação intestinal.

A relação entre fadiga e problemas de sono em pessoas com câncer é demonstrada em vários estudos (Davidson et al., 2002; Fiorentino \& Ancoli-Israel, 2006; Savard, Simard, Blanchet, Ivers, \& Morin, 2001). Ancoli-Israel, Moore e Jones (2001) apontaram que a fadiga é uma das principais queixas nesses pacientes, podendo ainda ocorrer uma possível relação com o tratamento oncológico (quimioterapia e radioterapia). Na presente pesquisa o elevado índice de fadiga relatado nas participantes do grupo clínico com má qualidade de sono apóia a idéia que esta queixa persiste após a conclusão do tratamento, o que é sugerido em pesquisas anteriores (Dow, Ferrell, \& Leigh, 1996; Hickok, Morrow, Roscoe, Mustian, \& Okunieff, 2005; Silva, 2009).

No presente estudo, a dor foi mais freqüente e intensa entre as participantes com má qualidade de sono. De fato, estudos sugerem que a dor e o sono mantêm influências mútuas: a dor repercute negativamente na qualidade de sono, e a privação de sono pode aumentar a sensibilidade à dor (Fortner et al., 2002; Sateia \& Lang, 2008).
Queixas psicológicas como ansiedade e depressão foram referidas com maior gravidade nas participantes com má qualidade de sono, corroborando estudos anteriores (Bardwell et al., 2008; Koopman et al., 2002; O‘Donnell, 2004; Palesh et al., 2007). Análises de regressão demonstraram que uma má qualidade de sono pode predizer uma pior qualidade de vida e maior gravidade de sintomas depressivos. Estudos longitudinais que investigam essas queixas nos mesmos participantes seriam úteis para identificar se tais condições já existiam durante o tratamento ou surgiram após o seu término, por outras condições. Pode-se especular que dada à quantidade de variáveis que operam na história de vida destes indivíduos, é improvável que um fator isolado seja responsável pela má qualidade de sono.

Os resultados referentes aos prejuízos nas questões sociais entre as mulheres com má qualidade de sono indicam que este tema deve ser mais detalhadamente explorado com a inclusão de medidas de rede de apoio social. Nesse contexto, Chor, Griep, Lopes e Faerstein (2001) mencionam as associações inversas entre dimensões de rede de apoio social e insônia.

Este estudo demonstrou que as participantes não tinham acessibilidade aos tratamentos não farmacológicos para insônia, uma vez que $40 \%$ das mulheres procuraram tratamento de cunho farmacológico. Embora intervenções psicológicas, em especial a terapia cognitivo-comportamental, sejam consideradas um dos tratamentos efetivos para problemas de sono em mulheres no pós-tratamento de câncer de mama (Fiorentino \& Ancoli-Israel, 2006; Haynes \& Bootzin, 2010), nenhuma participante relatou procurar esta modalidade de tratamento. Neste contexto, vale mencionar que os benefícios das terapias cognitivocomportamentais demonstram ser mais duradouros do que os do tratamento farmacológico (Cervena et al., 2004; Fiorentino \& Ancoli-Israel, 2006; Haynes \& Bootzi, 2010; Morin, 2004). O fato das participantes do presente estudo buscarem apenas o tratamento farmacológico pode ser conseqüência da falta de informação sobre outras modalidades de tratamento, como também a busca por alívio imediato. Sabe-se que a efetividade dos tratamentos cognitivo-comportamentais depende de uma análise funcional do contexto, modificação de estímulos ambientais e padrões comportamentais e reestruturação de crenças, enquanto que o farmacológico não (Ferreira, Soares, \& Pires, 2010). No entanto, para uma discussão mais aprofundada são necessárias pesquisas que investiguem fatores relacionados a opções por determinado tipo de tratamento.

A presente pesquisa evidenciou que a perturbação do sono é comum em mulheres após o tratamento oncológico, e entre aquelas que apresentam, existem diferenças de gravidade. Devido à variabilidade no desenvolvimento e na evolução da insônia, Fiorentino e Ancoli-Israel (2006) questionam os motivos pelos quais algumas mulheres com câncer de mama desenvolvem insônia durante e/ou após o tratamento enquanto outras não, quais seriam os fato- 
res envolvidos na sua gravidade e cronicidade, e também quais as características das pacientes que se beneficiam com tratamentos farmacológicos ou com terapias comportamentais. As autoras enfatizam que é provável que diferentes tipos de insônia (insônia primária ou comórbida) e sintomas advêm de variados fatores etiológicos que por sua vez irão interagir diferencialmente com os correlatos fisiológicos, comportamentais e sociais associados à experiência do câncer de mama. Conhecer a interação entre fatores biológicos, comportamentais e ambientais seria então fundamental no planejamento de intervenções eficazes voltadas para o aprimoramento do bem estar dessa população.

Este estudo avaliou a qualidade de sono por meio de instrumentos de auto-relato. Estudos posteriores poderiam utilizar em combinação com tais instrumentos, medidas objetivas do sono, como a actigrafia. $\mathrm{O}$ actígrafo é um instrumento equipado com um acelerômetro, que tem o tamanho e a forma de um relógio, e é usado no pulso para medir indiretamente a qualidade do sono por meio da quantificação e análise da atividade motora. Através da actigrafia é possível acessar medidas objetivas como (a) Tempo na Cama: duração, em minutos, entre o início (exemplo: ao apagar as luzes) e final do registro (exemplo: ao acender das luzes); (b) Latência para Início do Sono: tempo (minutos) entre o início do registro e o aparecimento do sono; (c) Período de Sono: duração (minutos) entre o início do sono até a última época de sono; (d) Despertares Intermitentes: quantidade, em minutos, de todas as épocas após o início do sono até o final do registro; (e) Porcentagem de Sono (eficiência do sono): Tempo efetivo de sono durante o tempo total na cama, que é calculado em porcentagem (Ferreira et al., 2010). Esta metodologia, junto aos instrumentos de auto-relato, seria útil para auxiliar as avaliações, fornecendo um maior detalhamento das características do sono nesta população.

Finalizando, a presente pesquisa contribuiu para a compreensão da insônia no contexto do pós-tratamento de câncer de mama ao mostrar que problemas de sono podem predizer aspectos de qualidade de vida e de bem estar psicológico. Espera-se que este trabalho possa estimular a produção de pesquisas nacionais sobre o tema, servindo como incentivo para o desenvolvimento de intervenções psicológicas para o manejo da insônia nesta população.

\section{Referências}

Ancoli-Israel, S., Moore, P., \& Jones, V. (2001). The relationship between fatigue and sleep in cancer patients: A review. European Journal of Cancer Care, 10, 245-255.

Bardwell, W. A., Profant, J., Casden, D. R., Dimsdale, J. E., Ancoli-Israel, S., Natarajan, L., et al. (2008). The relative importance of specific risk factors for insomnia in women treated for early-stage breast cancer. Psycho-Oncology, 17, 9-18.
Barichello, E., Sawada, N. O., Sonobe, H. M., \& Zago, M. M. F. (2009). Qualidade do sono em pacientes submetidos a cirurgia oncológica. Revista Latino-Americana de Enfermagem, 17(4), 481-488.

Beck, S. L., Berger, A. M., Barsevick, A. M., Wong, B., Stewart, K. A., \& Dudley, W. N. (2010). Sleep quality after initial chemotherapy for breast cancer. Support Care Cancer, 18(6), 679-689.

Beck, S. L., Schwartz, A. L., Towsley, G., Dudley, W., \& Barsevick, A. (2004). Psychometric evaluation of the Pittsburgh Sleep Quality Index in Cancer Patients. Journal of Pain and Symptom Management, 27(2), 140-148.

Bertolazi, A. N., Fagondes, S. C., Hoff, L. S., Dartora, E. G., Miozzo, I. C. S., Barba, M. E. F., et al. (2011). Validation of the Brazilian Portuguese version of the Pittsburgh Sleep Quality Index. Sleep Medicine, 12, 70-75.

Buysse, D. J., Angst, J., Gamma, A., Ajdacic, J., Eich, D., \& Rossler, W. (2008). Prevalence, course, and comorbidity of insomnia and depression in Young adults. Sleep, 31(4), 473-480.

Buysse, D. J., Hall, M. L., Strollo, P. J., Kamarck, T. W., Owens, J., Lee, L., et al. (2008). Relationships between the Pittsburgh Sleep Quality Index (PSQI), Epworth Sleepiness Scale (ESS), and clinical/polysomnographic measures in a community sample. Journal of Clinical Sleep Medicine, 4(6), 563-571.

Buysse, D. J., Reynolds, C. F., Monk, T. H., Berman, S. R., \& Kupfer, D. J. (1989). The Pittsburgh Sleep Quality Index: A new instrument for psychiatric practice and research. Psychiatric Research, 28(2), 193-213.

Campos, H. H., Bittencourt, L. R. A., Haidar, M. A., Tufik, S., \& Baracat, E. C. (2005). Prevalência de distúrbios do sono na pós-menopausa. Revista Brasileira de Ginecologia e Obstetricia, 27(12), 731-736.

Cardoso, H. C., Bueno, F. C. C., Mata, J. C., Alves, A. P. R., Jochims, I., Vaz Filho, I. H. R., et al. (2009). Avaliação da qualidade do sono em estudantes de medicina. Revista Brasileira de Avaliação Médica, 33(3), 349-355.

Carlson, L. E., Campbell, T. S., Garland, S. N., \& Grossman, P. (2007). Associations among salivary cortisol, melatonin, catecholamines, sleep quality and stress in women with breast cancer and healthy controls. Journal of Behavioral Medicine, 30(1), 45-58.

Carpenter, J. S., \& Andrykowski, M. A. (1998). Psychometric evaluation of the Pittsburgh Sleep Quality Index. Journal Psychosomatic Research, 45(1), 5-13.

Cervena, K., Dauvilliers, Y., Espa, F., Touchon, J., Matousek, M., Billiard, M., et al. (2004). Effect of cognitive behavioural therapy for insomnia on sleep architecture and sleep EEG power spectra in psychophysiological insomnia. Journal of Sleep Research, 13, 385-393.

Chor, D., Griep, R. H., Lopes, C. S., \& Faerstein, E. (2001). Medidas de rede e apoio social no Estudo Pró-Saúde: Prétestes e estudo piloto. Caderno de Saúde Pública, 17(4), 887-896.

Cohen, J. (1988). Statistical power analysis for the Behavioral Sciences. Hilsdale, NJ: Erlbaum.

Davidson, J. R., MacLean, A. W., Brundage, M. D., \& Schulze, K. (2002). Sleep disturbance in cancer patients. Social Science \& Medicine, 54, 1309-1321.

Dis, F. W., Mols, F., Vingerhoets, J. J. M., Ferrel, B., \& PollFranse, L. V. (2006). A validation study of the Dutch version of the Quality of Life - Cancer Survivor (QOL-CS) questionnaire in a group of prostate cancer survivors. Quality of Life Research, 15, 1607-1612. 
Rafihi-Ferreira, R., Pires, M. L. N. \& Soares, M. R. Z. (2012). Sono, Qualidade de Vida e Depressão em Mulheres no Pós-tratamento de Câncer de Mama.

Doi, Y., Minowa, M., Uchiyama, M., Okawa, M., Kim, K., Shibui, K., et al. (2000). Psychometric assessment of subjective sleep quality using the Japanese version of the Pittsburgh Sleep Quality Index (PSQI-J) in psychiatric disordered and control subjects. Psychiatry Research, 97, 165-172.

Dow, K. H., Ferrell, B. R., \& Leigh, S. I. (1996). An evaluation of the quality of life among long-term survivors of breast cancer. Breast Cancer Research and Treatment, 39, 261-273.

Dugan, W., McDonald, M. V., Passik, S. D., Rosenfeld, B. D., Theobald, D., \& Edgerton, S. (1998). Use of the Zung selfrating depression scale in cancer patients: Feasibility as a screening tool. Psycho-Oncology, 7, 483-493.

Eichling, P. S., \& Sahni, J. (2005). Menopause related sleep disorders. Journal of Clinical Sleep Medicine, 1(3), 291-300.

Ferreira, R. R., Soares, M. R. Z., \& Pires, M. L. N. (2010). De volta ao sono: Aspectos comportamentais e cognitivos da insônia. In M. M. C. Hubner, M. R. Garcia, P. R. Abreu, E. N. P. Cilo, \& P. B. Faleiros (Eds.), Sobre comportamento e cognição: Avanços recentes das aplicações comportamentais e cognitivas (pp. 75-84). Santo André, SP: ESETec.

Ferrell, B. R., Dow, K. H., \& Grant, M. (1995). Measurement of the quality of life in cancer survivors. Quality of Life Research, 4(6), 523-531.

Fiorentino, L., \& Ancoli-Israel, S. (2006). Insomnia and its treatment in women with breast cancer. Sleep Medicine Reviews, 10, 419-429.

Fleming, L., Gillespie, S., \& Espie, C. (2010). The development and impact of insomnia on cancer survivors: A qualitative analysis. Psycho-Oncology, 19, 991-996.

Fortner, B. V., Stepanski, E. J., Wang, S. C., Kasprowicz, S., \& Durrence, H. H. (2002). Sleep and quality of life in breast cancer patients. Journal of Pain and Symptom Management, 24(5), 471-480.

Furlani, R., \& Ceolim M. F. (2006). Qualidade do sono de mulheres portadoras de câncer ginecológico e mamário. Revista Latino-Americana de Enfermagem, 14(6), 872-878.

Haynes, P. L., \& Bootzin, R. R. (2010). Insomnia treatments: Moving from efficacy to effectiveness. Journal of Clinical Psychology, 66(11), 1131-1136.

Hickok, J. T., Morrow, G. R., Roscoe, J. A., Mustian, K., \& Okunieff, P. (2005). Occurrence, severity, and longitudinal course of twelve common symptons in 1129 consecutive patients during radiotherapy for cancer. Journal of Pain and Symptom Management, 30, 433-442.

Hindi, F., Dew, M. A., Albert, S. M., Lotrich, F. E., \& Reynolds, C. F. (2011). Preventing depression in later life: State of the art and science circa 2011. Psychiatric Clinics of North American, 34, 67-78.

Kleiboer, A., Bennett, F., Hodges, L., Walker, J., Thekkumpurath, P., \& Sharpe, M. (2011). The problems reported by cancer patients with major depression. Psycho-Oncology, 20, 62-68.

Koopman, C., Nouriani, B., Erickson, V., Anupindi, R., Butler, L. D., Bachmann M. H., et al. (2002). Sleep disturbances in women with metastatic breast cancer. The Breast Journal, 8 , $362-370$

Lustberg, L., \& Reynolds, C. F. (2000). Depression and insomnia: Questions of cause and effect. Sleep Medicine Reviews, 4(3), 253-362.

McChargue, D. E., Sankaranarayanan, J., Visovsky, C. G., Matthews, E. E., Highland, K. B., \& Berger, A. M. (2010). Predictors of adherence to a behovioral therapy sleep intervention during breast cancer chemotherapy. Support Care in Cancer, 1-8.
Morin, C. M. (2004). Cognitive-behavioral approaches to the treatment of insomnia. Journal of Clinical Psychiatry (Suppl. 16), 33-40

O'Donnell, J. F. (2004). Insomnia in cancer patients. Clinical Cornerstone, 6(Suppl. 1D), S6-S14.

Palesh, O. G., Collie, K., Batiuchok, D., Tilston, J., Koopman, C., Perlis, M. L, et al. (2007). A longitudinal study of depression, pain, and stress as predictors of sleep disturbance among women with metastatic breast cancer. Biological Psychology, 75, 37-44.

Passik, S. D. (2007). A survey of sleep problems in survivors of early stage breast cancer. Research project, Memorial Sloan-Kettering Cancer Center, New York, USA.

Passik, S. D., Kirsh, K. L., Donaghy, K. B., Theobald, D. E., Lundberg, J. C., Holtsclaw, E., et al. (2001). An attempt to employ the Zung Self-Rating Depression Scale as a "lab test" to trigger follow-up in ambulatory oncology clinics: Criterion validity and detection. Journal of Pain and Symptom Management, 21(4), 273-281.

Reich, M., Lesur, A., \& Perdrizet-Chevallier, C. (2008). Depression, quality of life and breast cancer: A review of literature. Breast Cancer Research and Treatment, 110, 9-17.

Rosekind, M. R., \& Gregory, K. B. (2010). Insomnia risks and costs: Health, safety, and quality of life. The American Journal of Managed Care, 16(8), 617-626.

Santos-Silva, R., Bittencourt, L. R. A., Pires, M. L. N., Mello, M. T., Taddei, J. A., Benedito-Silva, A. A., et al. (2010). Increasing trends of sleep complaints in the city of Sao Paulo, Brazil. Sleep Medicine, 11, 520-524.

Sateia, M. J., \& Lang, B. J. (2008). Sleep and cancer: Recent developments. Current Oncology Reports, 10, 309-318.

Savard, J., \& Morin, C. M. (2001). Insomnia in the context of cancer: A review of a neglected problem. Journal of Clinical Oncology, 19, 895-908.

Savard, J., Simard, S., Blanchet, J., Ivers, H., \& Morin, C. M. (2001). Prevalence, clinical characteristics, and risk factors for insomnia in the context of breast cancer. Sleep, 24(5), 583-590.

Silva, R. Z. M. (2009). Avaliação da fadiga em sobreviventes de câncer infantil e correlação com sintomas depressivos, distúrbios do sono e variáveis clínicas. (Dissertação de Mestrado não-publicada). Fundação Antônio Prudente, São Paulo, SP

Soares, C. N. (2006). Insônia na menopausa e perimenopausa - Características clínicas e opções terapêuticas. Revista de Psiquiatria Clínica, 33(2), 103-109.

Stepanski, E. J. (2006). Causes of insomnia. In T. Lee-Chiong (Ed.), Sleep: A comprehensive handbook (pp. 99-102). Hoboken, NJ: John Wiley \& Sons.

Zebrack, B. J., \& Chesler, M. A. (2001). A Psychometric analysis of quality of life cancer survivors (QOL-CS) in Survivors of Childhood Cancer. Quality of Life Research, 10, 319-329. 